\title{
Phylogenetic distinction of iNOS and IDO function in mesenchymal stem cell-mediated immunosuppression in mammalian species
}

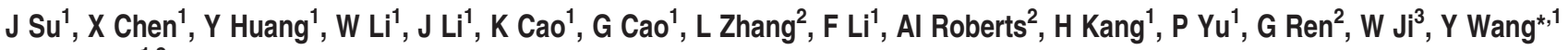 \\ and $Y \mathrm{Shi}^{*, 1,2}$
}

\begin{abstract}
Mammalian mesenchymal stem cells (MSCs) have been shown to be strongly immunosuppressive in both animal disease models and human clinical trials. We have reported that the key molecule mediating immunosuppression by MSCs is species dependent: indoleamine 2,3-dioxygenase (IDO) in human and inducible nitric oxide synthase (iNOS) in mouse. In the present study, we isolated MSCs from several mammalian species, each of a different genus, and investigated the involvement of IDO and iNOS during MSC-mediated immunosuppression. The characterization of MSCs from different species was by adherence to tissue culture plastic, morphology, specific marker expression, and differentiation potential. On the basis of the inducibility of IDO and iNOS by inflammatory cytokines in MSCs, the tested mammalian species fall into two distinct groups: IDO utilizers and iNOS utilizers. MSCs from monkey, pig, and human employ IDO to suppress immune responses, whereas MSCs from mouse, rat, rabbit, and hamster utilize iNOS. Interestingly, based on the limited number of species tested, the iNOS-utilizing species all belong to the phylogenetic clade, Glires. Although the evolutionary significance of this divergence is not known, we believe that this study provides critical guidance for choosing appropriate animal models for preclinical studies of MSCs.
\end{abstract}

Cell Death and Differentiation (2014) 21, 388-396; doi:10.1038/cdd.2013.149; published online 25 October 2013

Mesenchymal stem cells (MSCs) exist in almost all tissues and can be easily obtained from the bone marrow (BM), adipose tissues, and other tissues., ${ }^{1,2}$ MSCs were first identified, by Friedenstein and Caplan, as spindle-shaped cells with the capacity for self-renewal and multipotent differentiation. ${ }^{3}$ Given the lack of universal criteria for defining MSCs, a standard panel of surface markers is employed to define MSCs for basic research and clinical studies. Recently, MSCs have been found to be highly immunosuppressive. As such, they have been used in both animal models and clinical trials to treat various immune disorders, including graft versus host disease (GvHD), ${ }^{4,5}$ systemic lupus erythematosus (SLE), ${ }^{6}$ and rheumatoid arthritis. ${ }^{7}$

Notably, MSCs are not innately immunosuppressive but acquire this important function in response to the pro-inflammatory cytokines interferon- $\gamma$ or tumor necrosis factor- $\alpha$ in combination with interleukin (IL)- $1 \beta$. This process is orchestrated by chemokines, adhesion molecules and immunosuppressive molecules, and ultimately indoleamine 2,3-dioxygenase (IDO) in human, or inducible nitric oxide synthase (iNOS) in mouse. ${ }^{8,9}$ Immune cells are recruited by chemokines into the vicinity of MSCs where they are retained by adhesion molecules, and then suppressed either by locally high concentrations of nitric oxide (NO) or by IDO-mediated tryptophan depletion. ${ }^{10,11}$

Although MSCs have been proven to be very effective in inducing immunosuppression in both animal models and human trials, their reported effectiveness varies significantly. ${ }^{12-14}$ Given that the BM-derived MSCs from mouse differ in their immunomodulatory mechanism from that of humans, mouse models may be inappropriate for preclinical experiments to investigate the therapeutic effects of MSCs. Other animal models may better mimic human diseases, and also be more convenient than mouse models. In fact, immune disorder models established using rabbits, rats, pigs, and nonhuman primates can all be ameliorated or cured by MSC administration. ${ }^{15-19}$ The underlying mechanisms, however, remain to be elucidated.

To explore interspecies variation in the mechanisms of MSC-mediated immunosuppression, BM-MSCs were prepared from seven different species, including mouse, rat, hamster, rabbit, pig, monkey, and human. First, MSCs were defined by their morphology, marker expression, and differentiation ability. Then, their immunosuppressive capacity and

\footnotetext{
${ }^{1}$ Key Laboratory of Stem Cell Biology, Institute of Health Sciences, Shanghai Institutes for Biological Sciences, Chinese Academy of Sciences/Shanghai Jiao Tong University School of Medicine, 225 South Chongqing Road, Shanghai 200025, China; ${ }^{2}$ Child Health Institute of New Jersey, University of Medicine and Dentistry of New Jersey-Robert Wood Johnson Medical School, 89 French Street, NJ 08901, USA and ${ }^{3}$ Kunming Institute of Zoology, Chinese Academy of Sciences, Kunming 650223, China

*Corresponding author: Y Wang or Y Shi, Key Laboratory of Stem Cell Biology, Institute of Health Sciences, 225 South Chongqing Road, Shanghai 200025, China. Tel: + 86216384 8329; Fax: + 86216384 6467; E-mails: yingwang @sibs.ac.cn or yufangshi@ sibs.ac.cn Keywords: mesenchymal stem cells; inducible nitric oxide synthase; indoleamine 2; 3-dioxygenase; immunosuppression; mammalian phylogeny Abbreviations: MSCs, mesenchymal stem cells; NO, nitric oxide; iNOS, inducible nitric oxide synthase; IDO, indoleamine 2,3-dioxygenase; IL-1 $\beta$, interleukin-1beta; CFSE, carboxy fluorescein diacetate succinimidyl ester; L-NMMA, NG-monomethyl-L-arginine acetate salt; 1-MT, 1-methyl-tryptophan; PBMCs, peripheral blood mononuclear cells; Spl, splenocyte

Received 20.3.13; revised 20.8.13; accepted 02.9.13; Edited by RA Knight; published online 25.10.13
} 
the associated key molecules were investigated. We found that BM-MSCs isolated from the tested species conform to the current criteria for MSC identification; they appear fibroblast like, have the same surface markers, and can differentiate into adipocytes or osteoblasts. As expected, the immunosuppressive molecule that exerts the immunoregulatory function of BM-MSCs depended on the species. BM-MSCs derived from human, monkey, and pig utilized IDO for immunosuppression, whereas BM-MSCs from mouse, rat, hamster, and rabbit depended on iNOS expression. Interestingly, human, monkey, and pig are similarly positioned in the iNOS gene cladogram, whereas mouse, rat, hamster, and rabbit are all closely located in another branch. Therefore, our studies reveal not only the different molecules involved in MSCmediated immunosuppression in distinct species but also the potential relationships in the evolution of this difference.

\section{Results}

Derivation of BM-MSCs from different species. BM-MSCs from different species including human, monkey, pig, hamster, rabbit, mouse, and rat were obtained by adherent culture of BM cells, as described in Materials and Methods. All MSCs could be easily isolated and expanded to large-scale culture. They showed similar fibroblast-like cell morphology and formed homogenous colonies (Figure 1). Notably, MSCs derived from mouse and rat had to be passaged several times to achieve a high level of purity. For rabbit or pig BM-MSCs, non-adherent cells had to be removed at $24 \mathrm{~h}$ otherwise these cells would begin to adhere and thus contaminate MSCs. Although sharing the same morphology, BM-MSCs derived from human or monkey were bigger and had lower proliferation rates than other species. They required 7 days to achieve $90 \%$ confluence when started at $2 \times 10^{3}$ cells $/ \mathrm{cm}^{2}$, whereas mouse and rat BM-MSCs reached $90 \%$ confluence within only 2-3 days under similar plating densities. Therefore, although BM-MSCs from different species all had the similar spindle shape, they differed in cell size and growth rate.

Identification of BM-MSCs from different species. Because of a lack of universal criteria for defining MSCs, a panel looking at mRNA for surface markers was employed to exclude other cell types, and thereby identify them. At a minimum, MSCs should express stem cell markers like CD105, CD73, and CD29, and always be positive for CD44 and negative for CD45. ${ }^{20,21}$ As shown in Figure 2 and Table 1, BM-MSCs from the various species were remarkably consistent: human-, pig-, monkey-, rabbit-, and mousederived BM-MSCs all had the same phenotype: CD105 ${ }^{+}$ $\mathrm{CD}_{3}{ }^{+} \mathrm{CD} 2{ }^{+}{ }^{+} \mathrm{CD} 44^{+} \mathrm{CD}^{-} 5^{-}$; rat- and hamster-derived

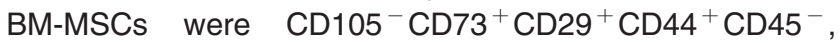
differing by their lack of CD105. Therefore, according to mRNA analyses of MSC surface markers, BM-MSCs derived



Figure 2 Surface markers of different species-derived BM-MSCs. All BM-MSCs were seeded in six-well plates and collected upon confluence for subsequent RNA extraction, RNA reverse transcription, and PCR assay. A panel of surface markers including CD105, CD73, CD29, CD44, and CD45 was detected for identification of BM-MSCs while GAPDH served as an input control



Figure 1 Morphology of BM-MSCs from different species. BM-MSCs before passage 10 from different species were isolated and maintained in DMEM complete medium as described. Their morphology was monitored under microscope and the scale bar indicates $100 \mu \mathrm{m}$ 
Table 1 Summary of MSC marker expression by BM-MSCs derived from different species

\begin{tabular}{lccccccc}
\hline & Human & Monkey & Pig & Mouse & Rat & Hamster & Rabbit \\
\hline CD 105 & + & + & + & + & - & - & + \\
CD 73 & + & + & + & + & + & + & + \\
CD 29 & + & + & + & + & + & + & + \\
CD 44 & + & + & + & + & + & + & + \\
CD 45 & - & - & - & - & - & - & - \\
\hline
\end{tabular}

from all seven species conform closely to the definition of MSCs.

\begin{abstract}
Multipotent differentiation potential of MSCs from different species. Besides the mRNA analyses for surface antigen expression profile, another criteria used to define MSCs is their potential to differentiate into adipocytes and osteoblasts. Our laboratory has established a robust system to induce adipocyte and osteoblast differentiation by human and mouse MSCs. Accordingly, BM-MSCs from human, mouse, and other species were induced to undergo adipogenesis or osteogenesis using the respective culture conditions, with successful differentiation checked by staining with oil red $\mathrm{O}$ or Alizarin Red $\mathrm{S}$, respectively. We found that BM-MSCs derived from all seven species could successfully differentiate into adipocytes (Figure 3a) or osteoblasts (Figure $3 b$ ), indicating that these BM-MSCs are indeed multipotent, further confirming their identity as MSCs.
\end{abstract}

Immunosuppression of BM-MSCs from different species. We have reported previously that the immunosuppressive function of MSCs is not intrinsic. ${ }^{10,22}$ Stimulation with pro-inflammatory cytokines is necessary to endow MSCs with the capacity to inhibit T-cell proliferation. The resultant immunosuppression is powerful; thus, MSCs are emerging as a promising tool in the treatment of immune disorders. ${ }^{23,24}$ Many laboratories have documented that the immunosuppressive effects of mouse MSCs on splenocyte (Spl) function is similar to that of human MSCs. ${ }^{23,25,26}$ This suggests that the same phenomenon may exist in many commonly used laboratory animals. To verify this, we tested the immunosuppressive function of MSCs derived from other five laboratory animals, including hamster, rabbit, rat, pig, and monkey. For each species, BM-MSCs were cocultured with stimulated Spls or peripheral blood mononuclear cells (PBMCs) at $1: 10$ ratio (MSC-to-Spl (or PBMC)) for 4 days. The proliferation of Spls or PBMCs was measured by the carboxyfluorescein diacetate succinimidyl ester (CFSE) dilution assay. As shown in Figure 4, MSCs from all tested species were found to be highly immunosuppressive to Spls or PBMCs. This suggests that it may be prudent to create more suitable animal models besides mouse so as to verify MSC-mediated immunosuppression in vivo.

\footnotetext{
Differential mechanisms account for similar immunosuppression by MSCs in distinct species. In our previous work, we have demonstrated the mechanisms underlying MSC-mediated immunosuppression. They are not innately immunosuppressive, but become so after exposure to pro-inflammatory cytokines. With sufficient cytokine stimulation,
}

MSCs can produce large amounts of chemokines, which recruit immune cells into their proximity. ${ }^{11}$ The recruited immune cells are then subject to the effects of locally high concentrations of immunosuppressive factors whose production is also induced by pro-inflammatory cytokines. Remarkably, the key molecule mediating this immunosuppression differs according to species; in mouse, it is NO produced by iNOS, whereas in human it is tryptophan depletion resulting from IDO upregulation. ${ }^{12}$ Yet, most researches to date have employed mouse models to verify the therapeutic effect of MSCs. As the mechanisms of MSC-mediated immunosuppression differ between mouse and human, we propose that other in vivo animal models, more suitable than mouse, should be used to mimic the immunosuppressive function of MSCs in human. To this end, we used specific inhibitors of iNOS and IDO to test whether MSCs from other species also employ either of these two molecules for immunosuppression. Accordingly, NG-monomethyl-L-arginine acetate salt (L-NMMA) or 1-methyl-tryptophan (1-MT), specific inhibitors of iNOS and IDO, respectively, were added to the MSC-Spl (or PBMC) coculture system. Interestingly, we found that immunosuppression by MSCs from rat, hamster, and rabbit was reversible by L-NMMA, but not 1-MT, indicating that they share a similar mechanism with mouse MSCs. Conversely, monkey and pig MSCs were dependent on IDO, making them similar to human MSCs (Figure 5a). The similar results were obtained when we measured the NO production in the supernatant of the MSC-Spl (or PBMC) cocultures. High level of NO concentration was detected in mouse, rat, hamster, and rabbit coculture system, whereas little NO was detected in human, monkey, and pig cocultures (Figure 5b). Therefore, our findings strongly suggest that animal models using monkey or pig would be more appropriate for preclinical studies of the effect of MSC-based therapy in human patients.

\section{Discussion}

MSCs exist in almost all tissues. They can be easily isolated from multiple tissues and expanded in large scale. Although previous studies have revealed multiple potential effector molecules in MSC-mediated immunosuppression, including IDO ${ }^{27}$ prostaglandin E2, ${ }^{28}$ transforming growth factor- $\beta 1,{ }^{29}$ $\mathrm{IL}-10,{ }^{30}$ heme oxygenase- $1,{ }^{31}$ hepatocyte growth factor, ${ }^{32}$ iNOS, ${ }^{23}$ IL-6, ${ }^{33}$ LIF, $^{34}$ galectins, and soluble HLA-G5, ${ }^{25,35}$ these studies focused mostly on either human MSCs or mouse MSCs. The key effector in rat- and monkey-derived MSCs also has been investigated to some extent: rat shares the same factor with mouse, whereas monkey is similar to human. ${ }^{12,36}$ The mechanisms in pig, hamster, and rabbit are not known. ${ }^{37,38}$ Therefore, the potential immunomodulatory mechanisms of MSCs from other species deserve further investigation, as some studies have revealed potent therapeutic effects by MSCs in vivo using these laboratory animals. Our study reveals that distinct mechanisms are involved in the immunosuppressive abilities of MSCs derived from several different species of laboratory animal, some dependent on iNOS and others on IDO. Notably, we have demonstrated that the key effector employed by MSCs from 
a



b



Figure 3 Adipocyte and osteoblast differentiation of BM-MSCs from different species. BM-MSCs before passage 12 were induced to differentiate into adipocytes and osteoblasts in differential medium and detected as described in Materials and Methods, untreated group used as control. (a) Adipocyte differentiation indicated by oil red 0 staining; the scale bar indicates $100 \mu \mathrm{m}$. (b) Osteoblast differentiation indicated by Alizarin red S staining; the scale bar indicates $500 \mu \mathrm{m}$

different species correlates with how closely they are related evolutionarily.

In addition to studies of the immunomodulatory effects of MSCs in vitro, many cases of successful MSC-based immunotherapy in mammalian species in vivo have been reported for conditions such as GvHD, ${ }^{4}$ liver disease, ${ }^{39,40}$ chronic spinal cord injury, ${ }^{41}$ and other diseases. Moreover, many MSC-based treatments have been tested in various animal models: mouse MSCs have been used to improve liver fibrosis ${ }^{42}$ and SLE; ${ }^{6}$ rat MSCs were reported to cure 
Parkinson's disease ${ }^{16}$ and liver cirrhosis; ${ }^{43}$ and rabbit MSCs were of benefit in treating experimental retinal holes. ${ }^{15}$ Finally, MSCs derived from monkey and pig were effective in

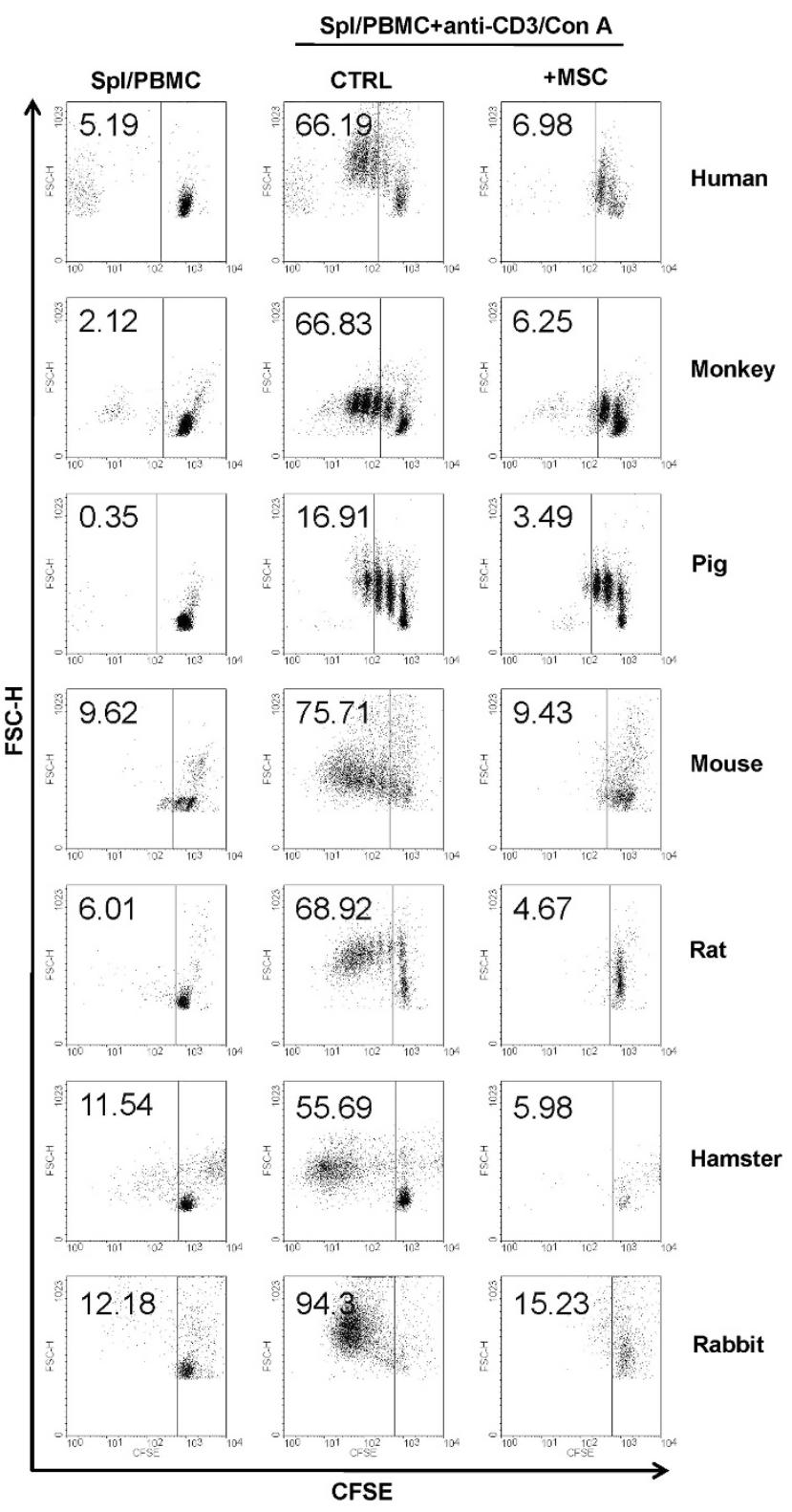

Figure 4 BM-MSCs isolated from different species have the same immunosuppression effects on Spls or PBMCs. PBMCs or Spls stained with CFSE were cocultured with BM-MSCs $\left(2.0 \times 10^{4}\right.$ cells per well in 96 -well plate $)$ at a ratio of $1: 10$ (MSC: T cell) for $96 \mathrm{~h}$, in the presence of conconavalin A (Con A) $(1 \mu \mathrm{g} / \mathrm{ml}$; for rat, rabbit, hamster, and pig), or anti-CD3 $(1 \mu \mathrm{g} / \mathrm{ml}$; for human, monkey, and mouse). CFSE fluorescence intensity reduction of PBMCs or Spls was detected by flow cytometry. Data are representative of three independent experiments peripheral nerve injury repair $^{19}$ and myocardial infarction treatment, ${ }^{44}$ respectively. Accordingly, MSCs have been proposed as a promising therapeutic strategy for disorders that benefit from their immunomodulation. Remarkably, MSCs from human and mouse exhibited distinct mechanisms of suppressing T-cell proliferation. In this study, we prepared BM-MSCs from human, monkey, pig, rabbit, hamster, rat, and mouse. These cells showed characteristics consistent with a series of universal standards for identifying MSCs: fibroblastlike morphology, the unique surface marker profile, $\mathrm{CD} 105^{+/-} \mathrm{CD} 73^{+} \mathrm{CD} 29^{+} \mathrm{CD} 44^{+} \mathrm{CD}^{-} 5^{-}$, and the ability to differentiate into adipocytes and osteoblasts. Experiments using specific inhibitors demonstrated that the effector molecules of MSC-mediated immunosuppression fall into two categories, mainly iNOS or mainly IDO, which is associated with the evolutionary relationships of the species.

Given that MSCs from different species employ distinct immunoregulatory molecules, we asked whether the divergent mechanisms could be ascribed to their different phylogenetic relationships. According to Carl von Linne's classification system, mouse, hamster, and rat are members of the taxonomic order rodentia. Notably, rabbit belongs to lagomorpha, which was formerly classified into rodentia. By contrast, human and monkey are primates, whereas pig belongs to artiodactyla. Recently, Song et al. ${ }^{45}$ verified this using phylogenomics and the multispecies coalescent model. An iNOS phylogenetic tree was constructed on the basis of amino-acid sequence of commonly used laboratory animals, including monkey, orangutan, pig, horse, cow, dog, mouse, rat, hamster, and rabbit (Figure 6) using the neighbor-joining method. ${ }^{46}$ From this iNOS phyletic tree, we found that current popular laboratory animals fall into one of five orders: primates, artiodactyla, carnivora, lagomorpha, or rodenti. All seven of the species we examined are located at a homologous clade that fits with the evolutionary genetics. This result strongly supports our hypothesis that species with the same mechanism of MSC-meditated immunosuppression are more closely related in evolutionary genetics.

As the use of MSC-based therapy for the treatment of disease is becoming generally accepted by both investigators and physicians, it is urgent to decipher which are the most suitable animal models for studying the clinical application of MSCs. Of course, we cannot determine empirically the mechanisms for each and every species of laboratory animal, but of the seven species examined here, we found that the mechanisms of MSC-mediated immunosuppression can be divided into two groups, one dependent on IDO and the other relying on iNOS. These two groupings seem to be similarly defined according to relationships in iNOS evolution, 47,48 based on our analysis of an iNOS phyletic tree. Therefore, the mechanism of MSC-mediated immunosuppression employed by a particular animal species can be estimated by its evolutionary relationship to other well-defined species.

Figure 5 BM-MSCs isolated from different species have different immunosuppression mechanism. (a) The immunosuppressive abilities of MSCs derived from different species. CFSE-stained PBMCs or Spls isolated from rat, rabbit, hamster, or pig were stimulated with conconavalin A (Con A) ( $1 \mu \mathrm{g} / \mathrm{ml})$, or from human, monkey, or mouse were stimulated with anti-CD3 $(1 \mu \mathrm{g} / \mathrm{ml})$ in the presence of corresponding BM-MSCs $\left(2.0 \times 10^{4}\right.$ cells per well in 96-well plate) at a ratio of $1: 10$ (MSC: T cell) for $96 \mathrm{~h}$. iNOS inhibitor L-NMMA (1 mM) or IDO inhibitor 1-MT (0.5 mM) were added into the coculture system. CFSE fluorescence intensity reduction of PBMCs or Spls was detected by flow cytometry. Data are representative of three independent experiments. (b) NO production in the MSC and activated Spls/PBMCs cocultures of different species was determined by Griess reagent 
a

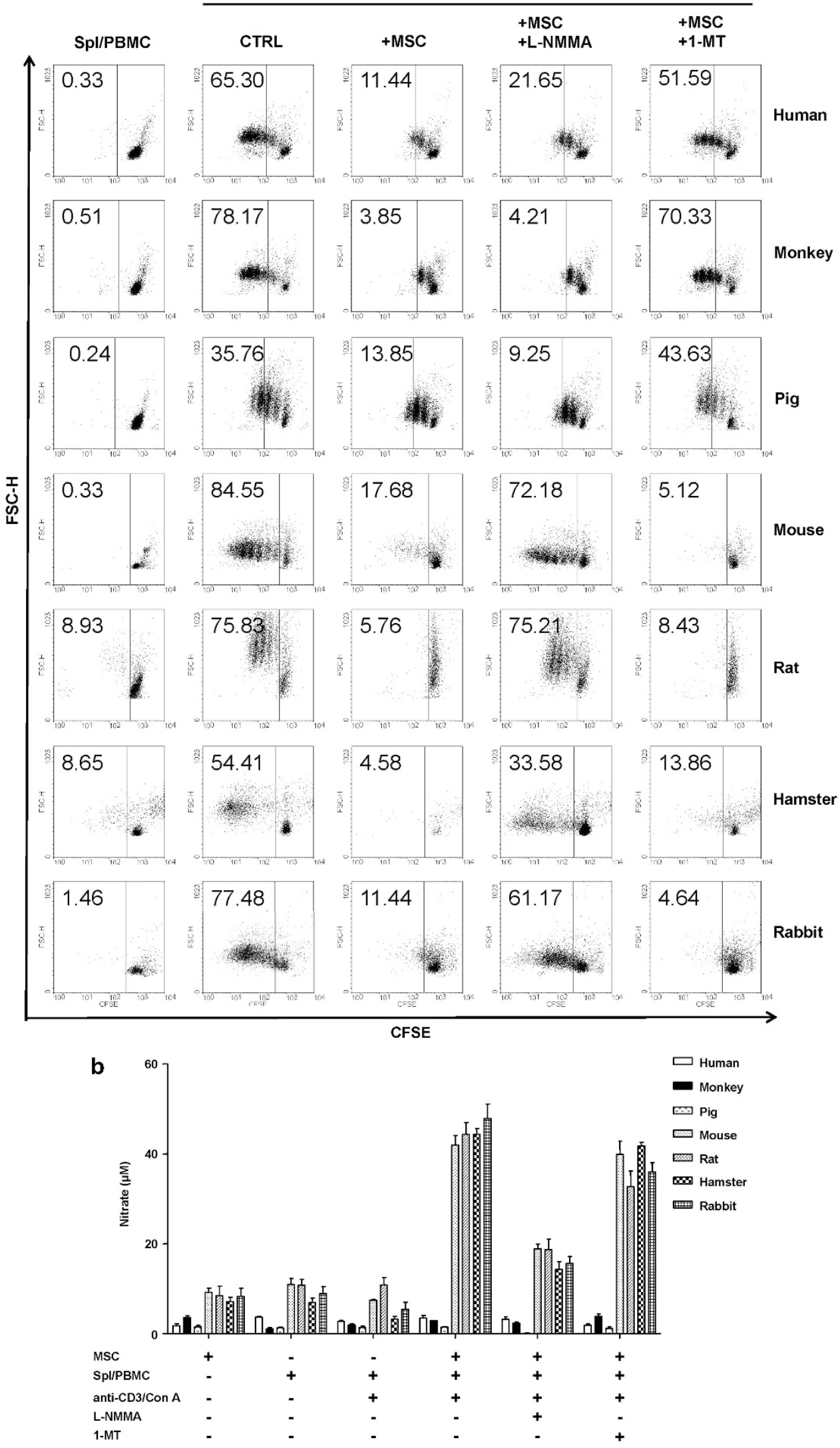


Utilization of the most appropriate animal models to predict the effects of MSC-based therapy in humans will be aided by this knowledge and lead to better and safer clinical use of MSCs.

\section{Materials and Methods}

Animals. BALB/c, C57BL/6 mice (Mus musculus), and Sprague-Dawley rats (Rattus norvegicus) were obtained from the Shanghai Laboratory Animal Center of the Chinese Academy of Sciences, Shanghai, China. New Zealand rabbits (Oryctolagus cuniculus) were from the Laboratory Animal Center of Chedun, Shanghai, China. Syrian hamsters (Mesocricetus auratus) were purchased from Vital River, Beijing, China. Rhesus macaques (Macaca mulatta) were from Kunming Institute of Zoology. All animals were maintained in the vivarium of Shanghai Jiao Tong University School of Medicine. The protocols used for animal experiments were approved by the Institutional Animal Care and Use Committee of the Institute of Health Sciences, Shanghai Institutes for Biological Sciences of the Chinese Academy of Sciences, and Shanghai Jiao Tong University School of Medicine.

Reagents. CFSE, indomethacin, dexamethasone, insulin, 3-isobutyl-1methylxanthine, L-ascorbic acids, $\beta$-glycerophosphate, alizarin red, oil red $\mathrm{O}$, formaldehyde solution, L-NMMA, 1-MT, and conconavalin A were purchased from



Figure 6 iNOS-based phylogenetic tree. The iNOS-coding region of amino-acid sequences of different species were used for evolutionary relationship alignment by neighbor-joining method. Numbers at nodes indicate the bootstrap support values. Domestic pig: Sus scrofa domesticus; bovine: Bos taurus; horse: Equus caballus; domestic dog: Canis lupus; rhesus macaques: Macaca mulatta; human: Homo sapiens; chimpanzee: Pan troglodytes; New Zealand rabbits: Oryctolagus cuniculus; Syrian hamsters: Mesocricetus auratus; BALB/c, C57BL/6 mice: Mus musculus; Sprague-Dawley rats: Rattus norvegicus
Sigma-Aldrich (St. Louis, MO, USA). Purified antibodies against human CD3 and mouse CD3 were purchased from eBioscience (La Jolla, CA, USA). Anti-monkey CD3 antibody was purchased from Abcam (Cambridge, MA, USA). Ficoll was purchased from GE Healthcare (Piscataway, NJ, USA). EZ-Sep Mouse 1X was purchased from Dakota Biotechnology (Shenzhen, China). Heat-inactivated fetal bovine serum (FBS), DMEM low-glucose medium, RPMI-1640 medium, trypsinEDTA, basic fibroblast growth factor (bFGF), glutamax, and penicillin-streptomycin solution were purchased from Invitrogen (Carlsbad, CA, USA).

Cells. Human MSCs were generated from human BM according to the approved protocol by the Institutional Review Board of Robert Wood Johnson Medical School. Human BM aspirates were diluted with equal parts RPMI-1640, overlaid on Ficoll, and centrifuged at $400 \times g$ for $30 \mathrm{~min}$ at $18-20^{\circ} \mathrm{C}$. Interface cells were collected, washed twice with RPMI-1640, and the cell pellet was resuspended in DMEM complete medium (DMEM low-glucose medium supplemented with $10 \%$ heat-inactivated FBS, $2 \mathrm{mM}$ glutamax, $100 \mathrm{U} / \mathrm{ml}$ penicillin, and $100 \mu \mathrm{g} / \mathrm{ml}$ streptomycin). Cells were cultured at $37^{\circ} \mathrm{C}$ in a humidified environment containing $5 \% \mathrm{CO}_{2}$. After $48 \mathrm{~h}$, non-adherent cells were washed away and supplied with fresh DMEM complete medium supplemented with bFGF ( $5 \mathrm{ng} / \mathrm{ml})$, with medium renewal every 3 days. Cells were collected and cryopreserved after confluence.

Monkey MSCs were obtained from rhesus monkeys BM according to the protocol by the Institutional Animal Care and Use Committee at Kunming Zoology Institute. To obtain BM from monkey, or pig, the animal was first anesthetized using amine $(30-50 \mathrm{mg} / \mathrm{kg})$, and then $2-5 \mathrm{ml}$ of BM was aspirated using a 12-gauge needle and $10 \mathrm{ml}$ heparinized syringe. For monkey and pig MSCs, non-adherent cells were removed after $24 \mathrm{~h}$ or $48 \mathrm{~h}$, respectively. Cells were then purified and cultured, as for human MSCs (above). Cells were passaged using 0.25\% trypsin-EDTA after reaching about $80 \%$ confluence.

BM from mouse, rat, and hamster was obtained from adult animals killed by cervical dislocation and from rabbits killed by air embolism. BM was flushed from the tibia and femur bones with $3 \times$ penicillin/streptomycin in phosphate-buffered saline (PBS). Unpurified cells were cultured without disturbance for $24 \mathrm{~h}$, then washed to remove non-adherent cells, and supplied with fresh DMEM complete medium supplemented with bFGF $(5 \mathrm{ng} / \mathrm{ml})$, with medium renewal every 3 days.

PBMCs from human, monkey, and pig were purified from fresh whole blood using ficoll density gradient centrifugation.

Mouse, rat, hamster, and rabbit Spls were obtained using EZ-Sep mouse density gradient centrifugation.

PCR reaction. The mRNA expression of specific cell surface markers was detected by reverse transcription-polymerase chain using specific primers. The primer sequences used for GAPDH, $\beta$-actin, CD105, CD73, CD29, CD44, and CD45 are listed (Table 2). Briefly, total RNA was extracted by RNAprep Pure Cell/Bacteria Kit (Tiangen Biotech, Beijing, China). First-strand CDNA synthesis was performed by 1 st cDNA Synthesis Kit (Tiangen Biotech). PCR was amplified by Takara rTaq (TaKaRa Biotech, Dalian, China). Target gene size was judged by 100-bp DNA ladder on agarose gel electrophoresis (TaKaRa Biotech).

Table 2 Primers for MSCs marker detection by PCR

\begin{tabular}{|c|c|c|c|c|c|}
\hline Primer & Forward primer $\left(5^{\prime}-3^{\prime}\right)$ & Reverse primer $\left(5^{\prime}-3^{\prime}\right)$ & Primer & Forward primer $\left(5^{\prime}-3^{\prime}\right)$ & Reverse primer $\left(5^{\prime}-3^{\prime}\right)$ \\
\hline $\begin{array}{l}\text { Human GAPDH } \\
\text { Human CD105 } \\
\text { Human CD73 } \\
\text { Human CD29 } \\
\text { Human CD44 } \\
\text { Human CD45 } \\
\text { Monkey GAPDH } \\
\text { Monkey CD105 } \\
\text { Monkey CD73 } \\
\text { Monkey CD29 } \\
\text { Monkey CD44 } \\
\text { Monkey CD45 } \\
\text { Pig GAPDH } \\
\text { Pig CD105 } \\
\text { Pig CD73 } \\
\text { Pig CD29 } \\
\text { Pig CD44 } \\
\text { Pig CD45 } \\
\text { Mouse GAPDH } \\
\text { Mouse CD105 } \\
\text { Mouse CD73 }\end{array}$ & $\begin{array}{l}\text { GAAGGTGAAGGTCGGAGT } \\
\text { ACGCTCCCTCTGGCTGTTG } \\
\text { CAGCGAGGATCCAGCAAG } \\
\text { GCATCCCTGAAAGTCCCAAG } \\
\text { GTGATGGCACCCGCTATG } \\
\text { AACAACCACTCTGAGCCCTTCT } \\
\text { ACTTCAACAGCGACACCCACTC } \\
\text { GTGGCATGGAAGTGACGG } \\
\text { CAGCGAGGACTCCAGCAAG } \\
\text { GCACCAGCCCATTTAGC } \\
\text { TCGCTACAGCATCTCTCGGAC } \\
\text { GGATCTCAGCAAACGGGAATA } \\
\text { AAGGTCGGAGTGAACGGATTT } \\
\text { AGTGCGACCTACAGCCTGTG } \\
\text { TGTTGGTAATGAAGTCGTGG } \\
\text { GATGAATGAAATGAGGAGGAT } \\
\text { CCACCTTCCGACTACTACGC } \\
\text { AACAAGGTGGATGTCTATGGCTA } \\
\text { TTCAATGGCACAGTCAAGGC } \\
\text { TTGAATGGCAACCACGAGC } \\
\text { GGCTGCTTCTCGCACTGA }\end{array}$ & $\begin{array}{l}\text { GAAGATGGTGATGGGATTTC } \\
\text { GCCCTTCGAGACCTGGCTAG } \\
\text { TATCCAACGATTCCCACAACT } \\
\text { CACTGTCCGCAGACGCACT } \\
\text { ACTGTCTTCGTCTGGGATGG } \\
\text { CCCCTGGTGGCACATCTAATA } \\
\text { CCCTGTTGCTGTAGCCAAATTC } \\
\text { TTGGGACGCAGGGCTAT } \\
\text { ATGGCATCGTAGCGTAGGG } \\
\text { GCCACCAAGTTCCCATC } \\
\text { CATACTGGGAGGTGTTGGACG } \\
\text { AGCTTTCTGTGGCACCAAGT } \\
\text { CATTTGATGTTGGCGGGAT } \\
\text { GCCAAGTGGAGTGGGATTC } \\
\text { GGAGGTGACTATGAATGGGTA } \\
\text { CCAGCAAAGTGAAACCCA } \\
\text { ATTGCCTGGGTTGTGCTTG } \\
\text { AGAAGGTTCACTGGGTGGGTC } \\
\text { TCACCCCATTTGATGTTAGCG } \\
\text { GAGCCTGACGGGAAACTGAT } \\
\text { CTGGTACTGGTCTCCGGC }\end{array}$ & $\begin{array}{l}\text { Mouse CD29 } \\
\text { Mouse CD44 } \\
\text { Mouse CD45 } \\
\text { Rat GAPDH } \\
\text { Rat CD105 } \\
\text { Rat CD73 } \\
\text { Rat CD29 } \\
\text { Rat CD44 } \\
\text { Rat CD45 } \\
\text { Rabbit GAPDH } \\
\text { Rabbit CD105 } \\
\text { Rabbit CD73 } \\
\text { Rabbit CD29 } \\
\text { Rabbit CD44 } \\
\text { Rabbit CD45 } \\
\text { Hamster } \beta \text {-actin } \\
\text { Hamster CD105 } \\
\text { Hamster CD73 } \\
\text { Hamster CD29 } \\
\text { Hamster CD44 } \\
\text { Hamster CD45 }\end{array}$ & $\begin{array}{l}\text { TGGTCAGCAACGCATATCT } \\
\text { CGTCCAACACCTCCCACTAT } \\
\text { GTTTTCGCTACATGACTGCACA } \\
\text { GCAAGTTCAACGGCACAG } \\
\text { CGGTTGTGATCTACAGCGTG } \\
\text { GCAGCCATCAAAGCAGACAT } \\
\text { GCCAACCAAGTGACATAGAGA } \\
\text { TGGCACAGCAGCAGATC } \\
\text { AACAACCGACGATGGACTGG } \\
\text { TTTGTGATGGGCGTGAACC } \\
\text { GCCAGCGTTGCGTCCTT } \\
\text { CTCCTTTCCTCTCAAATCCAG } \\
\text { AGAATGTCACCAACCGTAGCA } \\
\text { GGATGGCACCCGCTACA } \\
\text { TACTCTGCCTCCCGTTG } \\
\text { CCCATCTATGAGGGCTACGC } \\
\text { ACCGCACAGTGACCGTAAATG } \\
\text { ATCGTGGTGGGAGGACA } \\
\text { CTACTCCATGAAAGATGATCTGG } \\
\text { GGCGACTAGATTCCTCGGT } \\
\text { GTTACGTTGACATCCTTCCCTAT }\end{array}$ & $\begin{array}{l}\text { TTGTCCATCATTGGGTAAAAC } \\
\text { AGCCGCTGCTGACATCGT } \\
\text { AGGTTGTCCACTGACATCTTTC } \\
\text { CGCCAGTAGACTCCACGACA } \\
\text { CCCGAGTCTCAGTGCCATTT } \\
\text { AGCGGAGCCATTCAGGTAGA } \\
\text { TCAATAGGGTAGTCTTCAGCC } \\
\text { CTGCACAGATAGCGTTGG } \\
\text { GCCGTGAGTGTGGTGAGGTC } \\
\text { CCCTCCACAATGCCGAAGT } \\
\text { TGTTCTTCGGCGGGGTG } \\
\text { GTCCACGCCCTTCACTTTC } \\
\text { CACAAAGGAGCCAAACCCA } \\
\text { GGAGACCCACTGCTCACG } \\
\text { GCTGAGTGTCTGCGTGTC } \\
\text { CAGGAAGGAAGGCTGGAAAA } \\
\text { AAGGATGCTACAATGCTGGCG } \\
\text { GCTGTTCAGAAGAATGGGAT } \\
\text { GCAAAGTGAAACCCAGCAT } \\
\text { GTATCGTAGTGGGAGGTGTTG } \\
\text { GAAAGCATTAACCCTCCTTCG }\end{array}$ \\
\hline
\end{tabular}


Adipogenesis and osteogenesis. Adipocyte differentiation was achieved by culturing BM-MSCs to $90 \%$ confluence in adipocyte differentiation medium consisting of $0.5 \mathrm{mM}$ 3-isobutyl-1-methylxanthine, $60 \mu \mathrm{M}$ indomethacin, $10 \mathrm{nM}$ dexamethasone, $10 \mu \mathrm{g} / \mathrm{ml}$ insulin, and $2 \%$ FBS in DMEM complete medium, for 1-2 weeks, with medium renewal every 3 days. Oil red 0 staining solution was used to reveal lipid accumulations.

For osteoblast differentiation, BM-MSCs at $100 \%$ confluence were maintained in osteoblast differentiation medium (10 nM dexamethasone, $100 \mu \mathrm{M}$ L-ascorbic acids, $10 \mathrm{mM}$ $\beta$-glycerophosphate I, and 2\% FBS in DMEM complete medium ) for 3-4 weeks. Resulting calcium deposition was stained using $2 \%$ Alizarin Red S solution.

Proliferation assay. The proliferation of Spls or PBMCs was detected using CFSE staining and flow cytometry. Freshly isolated Spls or PBMCs $\left(<2 \times 10^{7}\right.$ cells) in $1 \mathrm{ml}$ of PBS containing $0.1 \%$ bovine serum albumin were stained with $5 \mu \mathrm{M}$ CFSE for $10 \mathrm{~min}$ in a $37^{\circ} \mathrm{C}$ water bath, with vortexing every $2 \mathrm{~min}$. The reaction was stopped by adding $0.5 \mathrm{ml}$ cold FBS. After 5 min, cells were washed with $5 \%$ FBS in PBS. Cells were then cocultured with MSCs at an MSC-to-Spl (or PBMC) ratio of 1:10. After 4 days, cells were collected and the remaining cell-associated CFSE fluorescence was analyzed by flow cytometry.

NO detection. The supernatant of MSCs and activated Spls/PBMCs cocultures was collected and stored at $-20^{\circ} \mathrm{C}$. NO concentration was assessed by determining the $\mathrm{NO}_{3}^{-}$and $\mathrm{NO}_{2}^{-}$levels using Griess reagent (modified; Sigma-Aldrich). $\mathrm{NaNO}_{2}$ served as a standard.

\section{Conflict of Interest}

The authors declare no conflict of interest.

Acknowledgements. This work was supported by grants from the Ministry of Science and Technology of China (2010CB945600, 2011DFA30630), Scientific Innovation Project of the Chinese Academy of Sciences (XDA01040107), International Cooperation and Exchanges NSFC (31010103908), Shanghai Municipal Key Projects of Basic Research (12JC1409200).

\section{Author Contributions}

JS contribute to conception and design, collection and assembly of data, data analysis and interpretation, and manuscript writing; WL, YH, and YW contributed to collection and assembly of data, data analysis and interpretation, and manuscript writing; XC, FY, KC, and GC performed data analysis and interpretation; AIR contributed to manuscript writing; WJ, GR, and LZ contributed to conception and design, data analysis and interpretation; JL, FL, and HK performed data collection; YS contributed to conception and design, manuscript writing, final approval of manuscript, and financial support.

1. Torensma R, Prins HJ, Schrama E, Verwiel ET, Martens AC, Roelofs H et al. The impact of cell source, culture methodology, culture location, and individual donors on gene expression profiles of bone marrow-derived and adipose-derived stromal cells. Stem Cells Dev 2012; 22: 1086-1096.

2. Pierro M, Ionescu L, Montemurro T, Vadivel A, Weissmann G, Oudit G et al. Short-term, long-term and paracrine effect of human umbilical cord-derived stem cells in lung injury prevention and repair in experimental bronchopulmonary dysplasia. Thorax 2012; 68 : 475-484

3. Caplan Al. Mesenchymal stem cells. J Orthop Res. 1991; 9: 641-650.

4. Kebriaei P, Isola L, Bahceci E, Holland K, Rowley S, McGuirk J et al. Adult human mesenchymal stem cells added to corticosteroid therapy for the treatment of acute graftversus-host disease. Biol Blood Marrow Transplant 2009; 15: 804-811.

5. Yanez R, Lamana ML, Garcia-Castro J, Colmenero I, Ramirez M, Bueren JA. Adipose tissuederived mesenchymal stem cells have in vivo immunosuppressive properties applicable for the control of the graft-versus-host disease. Stem Cells 2006; 24: 2582-2591.

6. Sun L, Akiyama K, Zhang H, Yamaza T, Hou Y, Zhao S et al. Mesenchymal stem cell transplantation reverses multiorgan dysfunction in systemic lupus erythematosus mice and humans. Stem Cells. 2009; 27: 1421-1432.

7. Papadopoulou A, Yiangou M, Athanasiou E, Zogas N, Kaloyannidis P, Batsis I et al. Mesenchymal stem cells are conditionally therapeutic in preclinical models of rheumatoid arthritis. Ann Rheum Dis 2012; 71: 1733-1740.

8. Shi Y, Hu G, Su J, Li W, Chen Q, Shou P et al. Mesenchymal stem cells: a new strategy for immunosuppression and tissue repair. Cell Res 2010; 20: 510-518.

9. Shi Y, Su J, Roberts Al, Shou P, Rabson AB, Ren G. How mesenchymal stem cells interact with tissue immune responses. Trends Immunol 2012; 33: 136-143.
10. Ren G, Zhang L, Zhao X, Xu G, Zhang Y, Roberts Al et al. Mesenchymal stem cellmediated immunosuppression occurs via concerted action of chemokines and nitric oxide. Cell Stem Cell 2008; 2: 141-150.

11. Li W, Ren G, Huang Y, Su J, Han Y, Li J et al. Mesenchymal stem cells: a double-edged sword in regulating immune responses. Cell Death Differ 2012; 19: 1505-1513.

12. Ren G, Su J, Zhang L, Zhao X, Ling W, L'Huillie A et al. Species variation in the mechanisms of mesenchymal stem cell-mediated immunosuppression. Stem Cells 2009; 27: 1954-1962.

13. Sato K, Ozaki K, Oh I, Meguro A, Hatanaka K, Nagai T et al. Nitric oxide plays a critical role in suppression of T-cell proliferation by mesenchymal stem cells. Blood 2007; 109: 228-234.

14. Ryan JM, Barry F, Murphy JM, Mahon BP. Interferon-gamma does not break, but promotes the immunosuppressive capacity of adult human mesenchymal stem cells. Clin Exp Immunol 2007; 149: 353-363.

15. Xuqian W, Kanghua L, Weihong $\mathrm{Y}, \mathrm{Xi} \mathrm{Y}$, Rongping D, Qin $\mathrm{H}$ et al. Intraocular transplantation of human adipose-derived mesenchymal stem cells in a rabbit model of experimental retinal holes. Ophthalmic Res 2011; 46: 199-207.

16. Danielyan L, Schafer R, von Ameln-Mayerhofer A, Bernhard F, Verleysdonk S, Buadze M et al. Therapeutic efficacy of intranasally delivered mesenchymal stem cells in a rat model of Parkinson disease. Rejuvenation Res 2011; 14: 3-16.

17. Chang $\mathrm{CH}$, Kuo TF, Lin FH, Wang JH, Hsu YM, Huang HT et al. Tissue engineering-based cartilage repair with mesenchymal stem cells in a porcine model. J Orthop Res 2011; 29: 1874-1880.

18. Sharma AK, Bury MI, Marks AJ, Fuller NJ, Meisner JW, Tapaskar N et al. A nonhuman primate model for urinary bladder regeneration using autologous sources of bone marrow-derived mesenchymal stem cells. Stem Cells 2011; 29: 241-250.

19. Hu J, Zhu QT, Liu XL, Xu YB, Zhu JK. Repair of extended peripheral nerve lesions in rhesus monkeys using acellular allogenic nerve grafts implanted with autologous mesenchymal stem cells. Exp Neurol 2007; 204: 658-666.

20. You Q, Cai L, Zheng J, Tong X, Zhang D, Zhang Y. Isolation of human mesenchymal stem cells from third-trimester amniotic fluid. Int J Gynaecol Obstet 2008; 103: 149-152.

21. Comite P, Cobianchi L, Avanzini MA, Zonta S, Mantelli M, Achille V et al. Isolation and ex vivo expansion of bone marrow-derived porcine mesenchymal stromal cells: potential for application in an experimental model of solid organ transplantation in large animals. Transplant Proc 2010; 42: 1341-1343.

22. Krampera M, Cosmi L, Angeli R, Pasini A, Liotta F, Andreini A et al. Role for interferongamma in the immunomodulatory activity of human bone marrow mesenchymal stem cells. Stem Cells 2006; 24: 386-398.

23. Xu G, Zhang L, Ren G, Yuan Z, Zhang Y, Zhao RC et al. Immunosuppressive properties of cloned bone marrow mesenchymal stem cells. Cell Res 2007; 17: 240-248.

24. Sudres M, Norol F, Trenado A, Gregoire S, Charlotte F, Levacher B et al. Bone marrow mesenchymal stem cells suppress lymphocyte proliferation in vitro but fail to prevent graftversus-host disease in mice. J Immunol 2006; 176: 7761-7767.

25. Gieseke F, Bohringer J, Bussolari R, Dominici M, Handgretinger R, Muller I. Human multipotent mesenchymal stromal cells use galectin-1 to inhibit immune effector cells. Blood 2010; 116: 3770-3779.

26. Hegyi B, Kudlik G, Monostori E, Uher F. Activated T-cells and pro-inflammatory cytokines differentially regulate prostaglandin E2 secretion by mesenchymal stem cells. Biochem Biophys Res Commun 2012; 419: 215-220.

27. Meisel R, Zibert A, Laryea M, Gobel U, Daubener W, Dilloo D. Human bone marrow stromal cells inhibit allogeneic T-cell responses by indoleamine 2,3-dioxygenase-mediated tryptophan degradation. Blood 2004; 103: 4619-4621.

28. Najar M, Raicevic G, Boufker HI, Fayyad Kazan H, De Bruyn C, Meuleman N et al. Mesenchymal stromal cells use PGE2 to modulate activation and proliferation of lymphocyte subsets: Combined comparison of adipose tissue, Wharton's Jelly and bone marrow sources. Cell Immunol 2010; 264: 171-179.

29. Groh ME, Maitra B, Szekely E, Koc ON. Human mesenchymal stem cells require monocyte-mediated activation to suppress alloreactive T cells. Exp Hematol 2005; 33 : 928-934.

30. Aggarwal S, Pittenger MF. Human mesenchymal stem cells modulate allogeneic immune cell responses. Blood 2005; 105: 1815-1822.

31. Chabannes D, Hill M, Merieau E, Rossignol J, Brion R, Soulillou JP et al. A role for heme oxygenase- 1 in the immunosuppressive effect of adult rat and human mesenchymal stem cells. Blood 2007; 110: 3691-3694.

32. Di Nicola M, Carlo-Stella C, Magni M, Milanesi M, Longoni PD, Matteucci P et al. Human bone marrow stromal cells suppress T-lymphocyte proliferation induced by cellular or nonspecific mitogenic stimuli. Blood 2002; 99: 3838-3843

33. Chan CK, Wu KH, Lee YS, Hwang SM, Lee MS, Liao SK et al. The comparison of interleukin 6-associated immunosuppressive effects of human ESCs, fetal-type MSCs, and adult-type MSCs. Transplantation 2012; 94: 132-138

34. Nasef A, Mazurier C, Bouchet S, Francois S, Chapel A, Thierry D et al. Leukemia inhibitory factor: Role in human mesenchymal stem cells mediated immunosuppression. Cell Immunol 2008; 253: 16-22.

35. Selmani Z, Naji A, Zidi I, Favier B, Gaiffe E, Obert L et al. Human leukocyte antigen-G5 secretion by human mesenchymal stem cells is required to suppress $T$ lymphocyte and natural killer function and to induce CD4 + CD25highFOXP3 + regulatory T cells. Stem Cells 2008; 26: 212-222. 
36. Zinocker S, Vaage JT. Rat mesenchymal stromal cells inhibit $\mathrm{T}$ cell proliferation but not cytokine production through inducible nitric oxide synthase. Front Immunol 2012; 3: 62 .

37. Noort WA, Oerlemans Ml, Rozemuller H, Feyen D, Jaksani S, Stecher D et al. Human versus porcine mesenchymal stromal cells: phenotype, differentiation potential, immunomodulation and cardiac improvement after transplantation. J Cell Mol Med 2012; 16: $1827-1839$.

38. Zhu F, Guo GH, Chen W, Peng Y, Xing JJ, Wang NY. Effect of bone marrow-derived mesenchymal stem cells transplantation on the inflammatory response and lung injury in rabbit with inhalation injury. Zhonghua Shao Shang Za Zhi 2010; 26: 360-365.

39. Houlihan DD, Hopkins LJ, Suresh SX, Armstrong MJ, Newsome PN. Autologous bone marrow mesenchymal stem cell transplantation in liver failure patients caused by hepatitis B: short-term and long-term outcomes. Hepatology 2011; 54: 1891-1892; author reply 1892.

40. Kharaziha P, Hellstrom PM, Noorinayer B, Farzaneh F, Aghajani K, Jafari $\mathrm{F}$ et al. Improvement of liver function in liver cirrhosis patients after autologous mesenchymal stem cell injection: a phase I-II clinical trial. Eur J Gastroenterol Hepatol 2009; 21 . 1199-1205.

41. Kishk NA, Gabr H, Hamdy S, Afifi L, Abokresha N, Mahmoud H et al. Case control series of intrathecal autologous bone marrow mesenchymal stem cell therapy for chronic spinal cord injury. Neurorehabil Neural Repair 2010; 24: 702-708.

42. Ishikawa H, Jo Jl, Tabata Y. Liver anti-fibrosis therapy with mesenchymal stem cells secreting hepatocyte growth factor. J Biomater Sci Polym Ed. 2012; 23: 2259-2272.
43. Pal R, Gopinath C, Rao NM, Banerjee P, Krishnamoorthy V, Venkataramana NK et al. Functional recovery after transplantation of bone marrow-derived human mesenchymal stromal cells in a rat model of spinal cord injury. Cytotherapy 2010; 12: 792-806.

44. Perin EC, Tian M, Marini FC 3rd, Silva GV, Zheng Y, Baimbridge F et al. Imaging long-term fate of intramyocardially implanted mesenchymal stem cells in a porcine myocardial infarction model. PLOS ONE 2011; 6: e22949.

45. Song S, Liu L, Edwards SV, Wu S. Resolving conflict in eutherian mammal phylogeny using phylogenomics and the multispecies coalescent model. Proc Natl Acad Sci USA 2012; 109: 14942-14947.

46. Saitou N, Nei M. The neighbor-joining method: a new method for reconstructing phylogenetic trees. Mol Biol Evol 1987; 4: 406-425.

47. Yuasa HJ, Takubo M, Takahashi A, Hasegawa T, Noma H, Suzuki T. Evolution of vertebrate indoleamine 2,3-dioxygenases. J Mol Evol 2007; 65: 705-714.

48. Ball HJ, Sanchez-Perez A, Weiser S, Austin CJ, Astelbauer F, Miu J et al. Characterization of an indoleamine 2,3-dioxygenase-like protein found in humans and mice. Gene 2007; 396: 203-213.

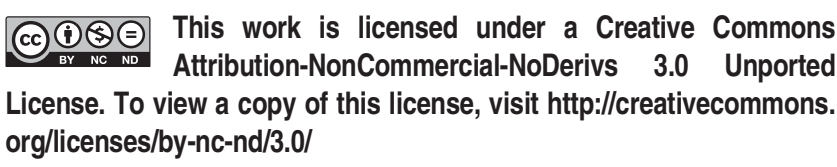

